\title{
Towards the Need Satisfaction in Gaming: A comparison of different gaming platforms
}

\author{
Anne-Flore Perrin, Touradj Ebrahimi \\ Multimedia Signal Processing Group \\ EPFL, Lausanne, Switzerland \\ \{anne-flore.perrin, touradj.ebrahimi\}@epfl.ch
}

\author{
Saman Zadtootaghaj \\ Telekom Innovation Laboratories \\ Deutsche Telekom AG ,Germany, \\ saman.zadtootaghaj@telekom.de
}

\author{
Steven Schmidt, Sebastian Möller \\ Quality and Usability Lab \\ TU Berlin, Germany, \\ \{steven.schmidt, sebastian.moeller\}@tu-berlin.de
}

\begin{abstract}
Recent advances in Virtual Reality (VR) technologies have resulted in a wider availability of Head Mounted Displays (HMDs). However, it is still unclear if VR gaming offers a substantial added value to players. For this reason a comparison of gaming experiences on VR HMD to those on mobile and PC, two other popular gaming platforms, is performed by conducting a user study via two games available on all three platforms. We explore the QoE of gaming by investigating momentous dimensions using the Player Experience of Need Satisfaction (PENS) questionnaire. The results show higher Presence and Autonomy obtained by using HMD when compared to the two other platforms. However, these factors alone did not improve the Overall Quality. To take advantage of the new technology, satisfaction of all psychological needs, especially Competency, must be assured.
\end{abstract}

\section{INTRODUCTION}

The fast development and placement on the market of various Head Mounted Displays (HMDs) raises the question of their real added value regarding gaming experience, when compared to typical gaming devices. In order to address this question, a user study investigating two games on three gaming platforms was carried out. Insights on how Player Experience of Need Satisfaction (PENS) questionnaire dimensions are impacted by the use of an HMD platform when compared to two other typical platforms, as well as the actual relevance of using such a questionnaire for gaming experience assessment, will be provided.

\section{Assessment of QuAlity of ExPERIEnCE}

In this study, the 18-items PENS questionnaire [1] was selected among widely used gaming and presence questionnaires, as it is the only one investigating Presence in addition to other known dimensions of Quality of Experience (QoE) in gaming. The questionnaire comprises five dimensions which are defined as follows: Competence refers to the intrinsic need to feel a sense of control and excellence during a gaming action; Autonomy represents to what extent gamers choices impact decisions and actions; Intuitive Control represents the degree of convenience provided by the set of commands allowing the conversion of gamers intentions into actions. Presence is the subjective feeling of being projected in an environment which is not the person's physical environment; Relatedness conveys the intrinsic desire to connect with others in a way that feels authentic and supportive. Since both games

QoMEX2017 - Erfurt, Germany; 978-1-5386-4024-1/17/\$31.00 (C)2017 IEEE under study are single-player, relatedness was not assessed. Instead, an additional question investigating the Overall Quality was included in the assessment procedure. This dimension is defined as "the outcome of an individual's comparison and judgment process." [2]. All questions are evaluated using a 7-point Likert Scale.

\section{STUDY DESIGN}

The mobile platform was a Samsung Galaxy S6 Edge smartphone, also used in combination with the Oculus Gear to constitute the HMD platform. The selection of the Oculus Gear as HMD platform was based on its high resolution and widespread usage due to its reasonable price. The Personal Computer (PC) platform was a 15-inch Mac Book Pro Retina. The test room provided a controlled, calm and neutral environment, fulfilling requirements in multimedia tests [3].

The two games, Deer Hunter (DH) and Gunship Battle (GB), were selected for the conducted experiment based on their availability on the three platforms and strong similarity across platforms (e.g. same game design, gameplay and level design). $\mathrm{DH}$ is a first-person shooting game which aims at hunting animals in the wild. GB is a flight simulator game in which the player is assigned missions to protect his military base or attack the enemy. Six 8-minute long gaming stimuli, resulting from the combination of games and platforms, were assessed individually in a within-participant design, using the questionnaire described in section II. The duration of a stimulus results from a trade-off between an adequate duration of gaming experience in order to get engaged and the constraint of limitation of in-game variations across subjects. The gaming scenario started with a tutorial of the controls followed by the first level of the game.

The test consisted of a training session prior to two test sessions. Each test session presented stimuli of one game on the three platforms. The order of stimuli were pseudorandomized to remove any platform-order bias. The subjects sample comprised 18 participants of 25.6 years old in average with a median age of 25.5 years old.

\section{RESUlts AND Discussion}

A one-way repeated measure Analysis of Variance (ANOVA), reported in Table I, was performed to determine statistically significant differences for the gaming platforms on the dependent variables (PENS dimensions and Overall 

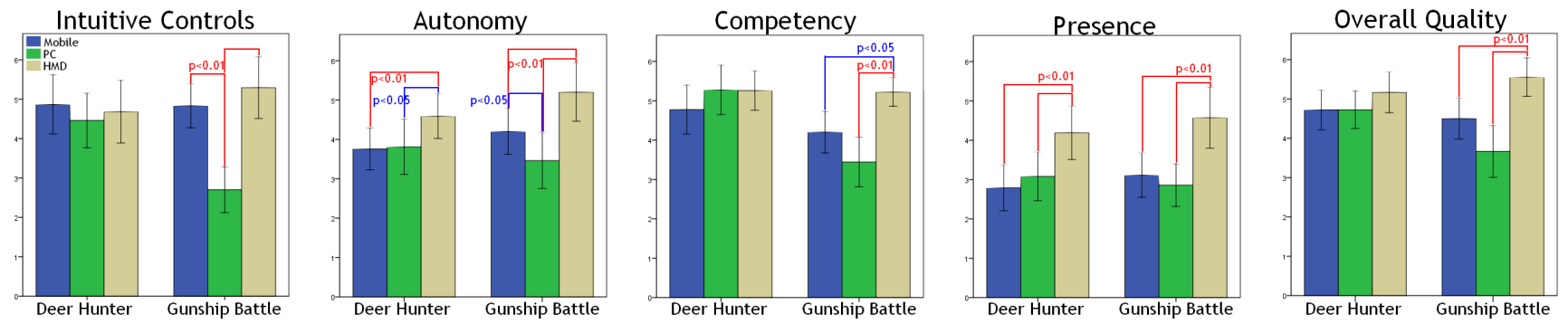

Fig. 1: Means and 95\% CIs of evaluated dimensions. Blue links, indicating $\mathrm{p}<0.05$, and red ones, $\mathrm{p}<0.01$, report the significant differences across platforms based on post-hoc tests [4].

TABLE I: ANOVA for the main effect of gaming platform.

\begin{tabular}{ccccccc} 
& \multicolumn{3}{c}{ Deer Hunter } & \multicolumn{3}{c}{ Gunship Battle } \\
\cline { 2 - 7 } & $\mathrm{F}$ & $\mathrm{P}$ & $\eta_{p}{ }^{2}$ & $\mathrm{~F}$ & $\mathrm{P}$ & $\eta_{p}{ }^{2}$ \\
\hline Presence & 30.53 & $\mathbf{0 . 0 1}$ & 0.64 & 34.81 & $\mathbf{0 . 0 1}$ & 0.67 \\
Intuitive Control & 0.43 & 0.65 & 0.03 & 36.33 & $\mathbf{0 . 0 1}$ & 0.68 \\
Competency & 2.2 & 0.12 & 0.12 & 14.93 & $\mathbf{0 . 0 1}$ & 0.47 \\
Autonomy & 10.1 & $\mathbf{0 . 0 1}$ & 0.37 & 18.22 & $\mathbf{0 . 0 1}$ & 0.52 \\
Overall Quality & 2.2 & 0.13 & 0.12 & 18.44 & $\mathbf{0 . 0 1}$ & 0.52 \\
\hline
\end{tabular}

Quality). The means and post-hoc test results of the assessed dimensions are graphically summarized in Fig. 1.

For DH, the ANOVA revealed a main effect of the used platform on Presence and Autonomy. A pairwise comparison of PC and Mobile showed no significant differences on any of the four PENS dimensions and the Overall Quality. For HMD, Presence and Autonomy were statistically significantly higher when compared to both other gaming platforms. However, this did not lead to higher ratings of Overall Quality and remaining dimensions. Additionally, Pearson's correlations between Overall Quality and Autonomy and Presence are 0.76 and 0.56, respectively. We can hereby conclude that most likely there is no causal relationship, or up to a moderate causal effect, between Presence and Autonomy with the Overall Quality for this specific game.

For GB, a main effect of the used platform on Presence and Autonomy was revealed as well. Ratings for HMD were significantly higher when compared to both other platforms. When comparing the results for the $\mathrm{PC}$ version, it has to be noted that the PC version was provided by an Android emulator. GB was not designed for the available input device on the PC, which led to poorer game controls for this platform. Consequently, Intuitive Control for PC was rated significantly lower than for the other platforms. However, no significant difference was observed between Mobile and HMD for Intuitive Control.

A comparison between PC and Mobile of the Overall Quality revealed no significant difference even though Intuitive Control and Autonomy of Mobile was superior compared to PC. It has to be noted that Competency was not rated significantly different for those two platforms. It appears that a violation of Intuitive Control and Autonomy does not necessarily affect the Overall Quality.

Furthermore, all PENS dimensions but Intuitive Control are statistically different for HMD compared to Mobile whereas they all are significant for the HMD versus PC comparison. Contrary to what was observed for the PC versus Mobile comparison, for HMD the Competency was significantly higher than for Mobile, even though Intuitive Control ratings were similar. However, the Overall Quality for HMD significantly increased compared to Mobile. Hereby we can conclude that for GB the likelihood of a casual relationship of Competency and the Overall Quality is high, since the change of the platform always led to a main effect on Competency and Overall Quality even when the influence on other dimensions was different. A possible reason why participants felt more competent using the HMD device is the increased view of the virtual environment as well as an increased accuracy when compared to the very small screen of the mobile phone.

\section{CONClusion}

This paper is a contribution to the ongoing research on the added value of virtual reality experience through newfangled affordable HMDs. The added value of three gaming platforms was investigated by comparing player experiences in two video games in terms of the influence dimensions Autonomy, Intuitive Control, Presence, and Competency as well as Overall Quality. From the outcome of our investigation we can conclude that the usage of a HMD increased Presence and Autonomy, for both games and potentially for other games, but it does not necessarily affect the Overall Quality. Presence might have a positive effect on Overall Quality, but its extent depends on additional requirements. However, for well-designed games such as GB, HMD can also offer additional advantages that lead to an increased Competency while playing. Our findings indicate that only when all psychological needs, in our case Autonomy and Competency, are satisfied, the use of an HMD can contribute to a richer experience with its superior feeling of Presence. Presence alone cannot compensate violations of those needs.

\section{ACKNOWLEDGMENT}

The work of German authors was supported by the German Research Foundation (DFG) within project MO 1038/21-1. Swiss authors acknowledge funding received from Swiss State Secretariat for Education, Research and Innovation (SERI). All authors acknowledge contributions from Marie SklodowskaCurie Action QoE-NET, grant agreement No 643072 


\section{REFERENCES}

[1] S. Rigby and R. Ryan, "The player experience of need satisfaction (pens): An applied model and methodology for understanding key components of the player experience," 2007.

[2] P. Le Callet, S. Möller, A. Perkis et al., "Qualinet white paper on definitions of quality of experience," European Network on Quality of Experience in Multimedia Systems and Services (COST Action IC 1003), vol. 3, 2012.

[3] ITU-T Recommendation P.911, "Subjective Audiovisual Quality Assessment Methods for Multimedia Applications. International Telecommunication," International Telecommunication Union, Geneva, 1998.

[4] R. A. Armstrong, "When to use the bonferroni correction," Ophthalmic and Physiological Optics, vol. 34, no. 5, pp. 502-508, 2014. 